Veritas E Scientia

Vol. 9. No 1

Enero - Junio del 2020

ISSN Edición Online: 2617-0639

https://doi.org/10.47796/ves.v9i1.282

\title{
MODELO DE ADMINISTRACIÓN DE CONTRATOS PARA MEJORAR LA GESTIÓN DE PROYECTOS EN OBRAS DE SANEAMIENTO
}

\author{
CONTRACT ADMINISTRATION MODEL FOR THE IMPROVEMENT OF SANITATION PROJECT \\ MANAGEMENT
}

\author{
Ulianov Farfán Kehuarucho ${ }^{1}$ \\ José Antonio Salgado Canal ${ }^{2}$
}

Aceptado: 09/03/2020

Publicado online: $10 / 07 / 2020$

\begin{abstract}
RESUMEN
Objetivo: Diseñar un modelo de administración de contratos para mejorar la gestión de proyectos en obras de saneamiento en la EPS TACNA S.A. todo ello desde la etapa de la licitación (convocatoria de una obra por la modalidad de contrata), pasando por la firma de contrato y la ejecución misma ya que se propone un modelo de contrato estandarizado para el buen manejo, control y seguimiento de los proyectos en ejecución.

Metodología: Se ha procedido al análisis de cada uno de los contratos firmados desde los años 2012 hasta la fecha que posterior se comparó con el contrato estandarizado FIDIC, además, se realizaron encuestas y entrevistas a expertos. Resultados: se desarrolló un modelo de administración de contrato estandarizado que permitirá gestionar un proyecto de saneamiento desde la etapa inicial de la licitación, buena pro, firma de contrato, ejecución y la etapa de cierre de proyecto. Conclusión: El presente estudio contempla todo el proceso de ejecución de la obra donde se identifican un sin número de consultas, contradicciones, omisiones, hasta negligencias; teniendo que ir dilucidando según el alcance del proyecto ya sea por la Supervisión o directamente por el Consultor Proyectista. Este confuso procedimiento genera riesgos en los proyectos que ejecuta la EPS Tacna S.A., que en muchos casos provocan que las inversiones se vean afectadas por paralizaciones, demoras injustificadas y finalmente hasta un arbitraje, debido a que las partes dentro del contrato de ejecución no aclararon muchos puntos que son sistemáticamente críticos y se vuelve recurrente en todos estos proyectos ejecutados que enfatiza e incide en una buena gestión de un proyecto, es en ese sentido se plantea un modelo de administración de contratos estandarizado que reducirá de forma significativa esta problemática.
\end{abstract}

Palabras claves: Contrato, cláusulas, FIDIC, estandarización, arbitraje

\footnotetext{
1'Maestro en Ingeniería Civil en Gerencia de la Construcción. EPS Tacna, S.A. Especialista en costos y presupuesto. ufarfan@epstacna.com.pe, ulianovfarfan@hotmail.com. ORCID:0000-0003-3855-1484

${ }^{2}$ Ingeniero Civil - UNI, magíster en Administración de Empresas MBA-ESAN, Certificado como PMP®, PRINCE $®$ y SFPC $\circledast$, es Director de proyectos múltiples de Infraestructura aplicando VDC/BIM/PM/LEAN/SCRUM. jsalgadoc@uni.edu.pe, jsalgadopmi@gmail.com. ORCID: 0000-0002-5298-0704
} 


\begin{abstract}
The research work was carried out with the information and evaluation of executed contracts for the execution of works in the Sanitation Services Provider Entity (EPS TACNA SA) Period 2012 - 2018, this investigation has the purpose of design a contract management model to improve the project management in sanitation works in the EPS TACNA SA; During this period of execution of works by CONTRACT MODE we have been able to see an innumerable numbers of queries, contradictions, omissions, negligence; having to be acquitted by the Supervision or directly by the Project Consultant. This confusing project generates risks in the projects executed by EPS Tacna SA, which in many cases cause the investments to be affected by paralyzes, unjustified delays finally in arbitration and this implies that they do not maintain their investment cycle and therefore loss of entity. FIDIC (International Federation of Consulting Engineers) is an international organization of standards for engineering and consulting construction best known by the family of FIDIC contract templates. This is how the new changes in the updating of the basic norms in the Peruvian State, they make every use of international recognized administrative standards, either as a basis for proposal or the writing of the final document. It is necessary to find a contract administration model to improve the project management in sanitation works so we need to plant a standard contract model that can control and overcome these shortcomings when they are executed in the EPS TACNA S.A. This research will yield a contract administration model that helps to manage a sanitation project from the initial lidding stage; good pro, contract signature, execution and the project closine is also proposed a standard contract model normative OSCE whit International regulations and contracts FIDIC, what will serve as the basis of solving the main problem.
\end{abstract}

Keywords: Contract, clauses, FIDIC, standardization, arbitration

\title{
INTRODUCCIÓN
}

La pregunta que surgió fue ¿Cómo mejoramos la administración de contratos para la gestión de proyectos en obras de saneamiento?; dado que, en la ejecución de obras por la modalidad CONTRATA se ha podido observar un sin número de consultas, contradicciones, omisiones, hasta negligencias; teniendo que ser absueltas por la Supervisión o directamente por el Consultor Proyectista. Este confuso procedimiento genera riesgos en los proyectos que ejecuta la EPS Tacna S.A., que en muchos casos provocan que las inversiones se vean afectadas por paralizaciones, demoras injustificadas finalmente en arbitraje, esto conlleva a que no cumpla su ciclo de inversión y por ende pérdidas para la entidad. EI FIDIC (Federación Internacional de Ingenieros Consultores) es una organización internacional de estándares para la ingeniería y construcción de consultoría mejor conocida por la familia de plantillas de contratos FIDIC. Es así que los nuevos cambios en actualizaciones de normativa en el Estado Peruano, hacen cada vez más el normal uso de estándares administrativos reconocidos internacionalmente ya sea como base de propuesta o la redacción del documento definitivo. 
Ante este problema, es necesario hallar un modelo de administración de contrato para mejorar la gestión de proyectos en obras de saneamiento y por ende plantear un modelo de contrato estandarizado que pueda controlar y superar estas carencias cuando se ejecutan las obras en la EPS Tacna S.A.

La actividad principal de la EPS Tacna S.A. es la prestación de los servicios de saneamiento, los cuales están comprendidos por los servicios de agua potable y alcantarillado sanitario, con más de 61,000 usuarios con conexiones de agua y alcantarillado. Asimismo, dentro de sus competencias están las de dotar agua de calidad, operar y mantener el sistema de alcantarillado y el tratamiento de las aguas servidas mediante infraestructura sanitaria adecuada (EPS TACNA S.A., 2015). A la fecha la Gerencia de Ingeniería como Unidad Ejecutora de Inversión dentro del marco normativo vigente INVERTE.PE, ejecuta Proyectos de Inversión bajo la modalidad de contrato Indirecto (contrata), utilizando la plataforma de procesos de selección Nacional de Estado SEACE (concurso público), es así, que una vez finalizado este procedimiento los proyectos de inversión se materializan con la elaboración del CONTRATO DE OBRA, para iniciar el procedimiento netamente de Ejecución de Obra Física. Una vez iniciado el proceso de ejecución de la obra se identifican un sin número de consultas, contradicciones, omisiones, hasta negligencias; teniendo que ir dilucidando según el alcance del proyecto ya sea por la Supervisión o directamente por el Consultor Proyectista. Este confuso procedimiento genera riesgos en los proyectos que ejecuta la EPS Tacna S.A., que en muchos casos provocan que las inversiones se vean afectadas por paralizaciones, demoras injustificadas y finalmente hasta un arbitraje, debido a que las partes dentro del contrato de ejecución no aclararon muchos puntos que son sistemáticamente críticos y se vuelve recurrente en todos estos proyectos ejecutados que enfatiza e incide en una buena gestión de un proyecto.

Por otra parte, a la fecha, la Entidad no cuenta con la elaboración legal y técnica de contratos que puedan minimizar estos riesgos y por ende se cumpla la ejecución técnica del proyecto contractualmente, razón por la cual se tienen identificadas entre otras dificultades de obra como: Inaplicación de penalidades por incumplimiento de plazo contractual, suscripción de contrato de forma irregular y/o sin documentación reglamentaria, Irregularidades en la contratación de los servicios de consultoría, ejecución de obras, deficiencias en resolución de contrato y liquidación de obra, no renovación de cartas fianzas, obras defectuosas, inconclusas o inoperativas y aprobación irregular de expedientes de adicionales de obra.

Bajo la premisa descrita en los párrafos anteriores una vez iniciada la ejecución de la obra existe innumerables situaciones e inconvenientes, poniendo en riesgo la inversión y el objetivo del contrato que es la culminación y ejecución de la obra misma. Lo anterior, aunado a otros problemas propios de América Latina, como la corrupción, el desvío de recursos para campañas políticas, etc., (problemas que existen en todo el mundo, pero en nuestro lado del mundo son más visibles) han hecho que el sector de la construcción necesite urgentemente de nuevos mecanismos que coadyuven a mejorar sus condiciones actuales, (SANCHEZ, 2017).

La industria de la construcción tiene un importante impacto en la vida diaria de las personas, ya que las obras como escuelas, hospitales y carreteras han permitido la adaptación de los seres humanos a distintos climas y mejores condiciones para desarrollarse en todos los ámbitos. La realización de estos proyectos requiere del apoyo de consultores expertos en la realización de contratos de construcción, que conozcan el sector, sus necesidades y, sobre todo, las características que lo distinguen de otro tipo de proyectos, previendo sus problemáticas y posibles soluciones a las mismas. 
En el caso de Europa y Estados Unidos, cada vez es más extendido el uso de los contratos desarrollados por la Federación Internacional de Ingenieros Consultores (FIDIC por sus siglas en francés), que al ser estandarizados con base en las necesidades del sector que nos ocupa, dotan de certeza jurídica a las partes que firman un contrato de obra (SANCHEZ, 2017).

En estos últimos años existe una fuerte demanda de obras de saneamiento ya que la Política del Estado Peruano enmarcó sus proyectos hacia el cierre de brechas priorizando los proyectos de servicios públicos en las necesidades básicas, mapa de pobreza, SISFOH, áreas geográficas, etc. esto implica de manera considerable el incremento dentro de las prelaciones en todas las entidades la ejecución de obras de saneamiento asimismo, cumplir aspectos como definir, mostrar y sustentar la pertinencia del proyecto. (MINISTERIO DE ECONOMIA Y FINANZAS, 2015). El Reglamento aprobado mediante Decreto Supremo No. 184-2008-EF La Ley y su Reglamento entraron en vigencia a partir del 01 de febrero del 2009, Esta normativa (LEY DE CONTRATACIONES DEL ESTADO, 2009), se creó con la finalidad de adecuarnos a estándares internacionales, como fue la implementación del Tratado de Libre Comercio con los Estados Unidos de Norteamérica, con la finalidad de consolidar el crecimiento e intercambio económico. Esta normativa es importante para nuestro sistema de contratación estatal a nivel histórico, porque exige un esfuerzo conjunto por parte de los operadores jurídicos, como funcionarios, proveedores, fiscalizadores, entre otros, porque favorece a la gestión eficiente. Por medio de esta normativa se creó el OSCE en reemplazo del CONSUCODE, las características de esta última entidad son gerencialista y burocrática. Asimismo, La normativa actual de contrataciones del Estado y el OSCE, fueron concebidos con los actuales pensamientos administrativos, como el de calidad total, mejoramiento continuo, calidad de servicio y la reingeniería. (PEREIRA, I. QUIJADA, V. PACOMPEA G. y LEGUA, M , 2010)

Los modelos contractuales estandarizados emergen como un uso del comercio en el ámbito de la ingeniería y construcción, pues se trata de uno de los sectores de la industria que más ha desarrollado esta práctica; pero además la utilización de estos modelos se encuentra reforzado con un proceso de interpretación sistemático de dichos contratos por parte de la actividad arbitral, y en especial, de la proveniente de instituciones como la Cámara de Comercio Internacional y la Corte de Arbitraje Internacional de Londres, (RODRIGUEZ, M, 2006, pág. 9) Por otro lado, a nivel mundial la industria de la construcción una de sus preocupaciones fue la de unificar criterios y es así que cada vez hicieron intentos de crear contratos modelos. La Comisión de la Naciones Unidas para el Derecho Mercantil Internacional (CNUDMI o UNCITRAL) elaboró la llamada "Guía Jurídica de la CNUDMI para la redacción de Contratos Internacionales de Construcción de Obras Industriales"; estandarización generadora de un nuevo e importante orden regulador para el sector de la construcción. (THE JOINTS CONTRACTS TRIBUNAL , 2014) En el Reino Unido se viene utilizando más de cinco formatos: El modelo ICE7; la JCT 98; la IFC 98; la MW 98 y fundamentalmente los formatos NEC New Engineering Contracts. Este proceso de estandarización se fortaleció gracias a la participación activa del Joint Contracts Tribunal (JCT), ha producido formas contractuales estándar, guías y otros documentos de uso general en la industria de la construcción, así como del Technology and Construction Court (TCC) (RODRIGUEZ, M, 2006, págs. 11-12). En el Japón el proceso de estandarización tiene un desarrollo significativo debido a la labor de la Engineering Advancement Association of Japan. Esta institución ha publicado una serie de modelos contractuales que viene teniendo gran influencia a nivel internacional. Entre estos modelos tenemos el ENAA Model Form-International Contract for Process Plant Construction (Turnkey Lump Sum Basis) (RODRIGUEZ, M, 2006, pág. 12). En determinados 
países como Arabia Saudita y Nigeria (PAREDES, G y GRAY, J, 2015), por ejemplo, el proceso de estandarización actualmente se basa en modelos creados por el Fondo Europeo de Desarrollo, más conocidos por la abreviatura de FED, marco importante de reglas también conocidas internacionalmente, así como en otros modelos de contratos internacionales como los FIDIC. Sin embargo, los formatos contractuales más utilizados a nivel internacional son los modelos publicados por la FIDIC, la agremiación profesional de la ingeniería y construcción más prestigiosa del mundo; $y$, dentro del amplio rango de especialidades - a semejanza de un arco iris- es el Libro Rojo el más conocido y utilizado a nivel internacional. El Libro Rojo es aplicado para la ejecución de obras civiles de construcción con diseño elaborado por el propietario o comitente.

De los anteriores párrafos descritos se puede finalizar que estos constantes aportes, cambios y modificaciones en la búsqueda del perfeccionamiento y la estandarización de los contratos nacionales e internacionales son con el único propósito de tener un contrato de obra donde explícitamente contemplen dentro de sus cláusulas aspectos legales y técnicos para evitar los conflictos posteriores. Bajo ese contexto es que los países con alta industrialización en la construcción investigan y estandarizan procedimientos obteniendo resultados óptimos y en aras de continuar y aplicar estos procedimientos ésta investigación va a proponer un modelo de administración de contrato estandarizado que servirá para mejorar la gestión de proyectos en obras de saneamiento en la EPS TACNA S.A., asimismo, menguará los problemas que a la fecha agudizan el proceso de ejecución de las obras de saneamiento y llegan a procedimientos tediosos, multas, costos sobrevalorados y terminan en arbitrajes que demoran muchos años en solucionar hasta alcanzar un laudo arbitral.

La Administración Pública en el Perú, hace notar que, en nuestro ordenamiento, a diferencia de otros países de nuestro entorno, como el colombiano o el argentino, no existe una legislación unitaria que aglutine la regulación de toda la contratación estatal. (DANOS, J, 2006)

\section{OBJETIVOS}

- Diseñar un modelo de administración de contratos para mejorar la gestión de proyectos en obras de saneamiento en la EPS TACNA S.A.

- Identificar las potenciales controversias durante la ejecución en proyectos de saneamiento.

- Desarrollar un modelo de contrato para obras de saneamiento estandarizado a un modelo internacional.

\section{METODOLOLOGÍA}

El tipo de investigación fue aplicada, puesto que se buscó solucionar un problema, de nivel descriptivo y busca analizar el comportamiento de las variables de estudio buscando la generación de conocimiento a través de del desarrollo de un modelo de administración de contrato estandarizado. El nivel de la investigación es Propositiva, orientado a la innovación, el cual implica desarrollar un modelo de administración de contrato estandarizado. El estudio se desarrolló en la Entidad Prestadora de Servicios de Saneamiento EPS TACNA S.A. en coordinación con la Gerencia de Administración para acceder a los archivos de los contratos, en aproximadamente 10 meses.

Para la presente investigación se consideró como población los contratos (7 contratos) elaborados durante el tiempo de intervención por la EPS TACNA S.A. La muestra del estudio 
fue de revisión exhaustiva a los contratos ejecutados y en proceso de ejecución. Variables Independientes: Desarrollo de un modelo de administración de contrato estandarizado al contrato internacional FIDIC (Número de formatos, número de procesos e implementación). Variables Dependientes: Mejorar las controversias, posibles problemas en la ejecución de proyectos a causa de Contratos ambiguos o carencia de cláusulas aclaratorias (Cantidad de controversias, cantidad de arbitrajes, disminución de conflictos, disminución de controversias y disminución de ampliaciones de plazo.

Durante el proceso de contratación corresponderá tener muy en cuenta que se necesita de documentos de entrada que deberán ser revisados exhaustivamente la concordancia del objeto de contratación versus el expediente técnico aprobado. Uno de los principales problemas que acarrea la entidad, es que los contratos de obra que es el documento ya perfeccionado luego del proceso de selección, carecen de cláusulas que nos ayuden a tener una buena administración del contrato en la etapa de ejecución, esto ocasiona muchos problemas que gracias a las encuestas realizadas nos vislumbran para así dar una alternativa de solución ante tal hecho. Una vez realizada, seleccionada y corregida las preguntas que consigna la encuesta, se tuvo la participación de 20 encuestados, cuya población son profesionales involucrados en lo que respecta a la administración de Proyectos de Saneamiento y que por su experiencia conocen la problemática de cerca y procedieron a contestar con preguntas cerradas que luego se procedió a consolidar en tabla de datos e interpretación que se muestran.

\section{RESULTADOS DE LA ENCUESTA}

El 100\% de los profesionales encuestados son ingenieros y eso favorece el criterio que pueden dar en sus respuestas referido a sus necesidades insatisfechas en materias de modelos, gerenciamiento, formatología control, entre otros temas similares. El 10\% de los profesionales encuestados se encuentra en el rango máximo de 3 años de experiencia involucrados en proyectos de saneamiento, concluyéndose con un $55 \%$ de profesionales que se encuentran en más de los 15 años de experiencia profesional. El 100\% de los profesionales encuestados si han participado en la ejecucion de una obra de saneamiento en el sector publico, esto permite y garantiza el amplio conocimiento que se cuenta. El $45 \%$ de los profesionales encuestados ejecutaron obras entre 1 millón a 5 millones mientras que el $40 \%$ ejecutaron obras entre los 5 millones a 20 millones.

Tabla 1

Problema más frecuente que ha presenciado en la administración de contratos en obras de saneamiento

\begin{tabular}{|c|c|c|}
\hline \multicolumn{2}{|c|}{ VRECUENCIA } & RELEVANCIA \\
\hline Clausulas ambiguas & 45 & $9 \%$ \\
\hline Clausulas de contrato implícitas & 45 & $9 \%$ \\
\hline Inaplicación de penalidades & 64 & $13 \%$ \\
\hline No renovación de cartas fianza & 51 & $11 \%$ \\
\hline TDR imprecisos & 72 & $15 \%$ \\
\hline Obras inconclusas & 55 & $12 \%$ \\
\hline Obras defectuosas & 51 & $11 \%$ \\
\hline Fallas y errores de tipeo & 42 & $9 \%$ \\
\hline contrato & 51 & $11 \%$ \\
\hline TOTAL & 476 & $100 \%$ \\
\hline
\end{tabular}

Fuente: Elaboración propia 
La tabla 1 muestra los problemas más frecuentes relevantes son TDR imprecisos, inaplicación de penalidades, obras inconclusas, no renovación de cartas de fianzas, obras defectuosas, sus valores fluctúan entre los 11 al 15\% y otros parámetros están en $9 \%$, del grafico se aprecia que la mayoría de los problemas tienen incidencia de uno u otra forma en el proceso.

Tabla 2

Relevancias de un contrato donde no estén bien definidas las cláusulas

\begin{tabular}{|c|c|c|}
\hline VALIDO & FRECUENCIA & RELEVANCIA \\
\hline Arbitrajes & 118 & $21 \%$ \\
\hline ampliación de plazo & 98 & $17 \%$ \\
\hline incremento de presupuesto & 86 & $15 \%$ \\
\hline mayores pagos de GG & 82 & $15 \%$ \\
\hline Obras defectuosas & 95 & $17 \%$ \\
\hline Inaplicación de penalidades & 86 & $15 \%$ \\
\hline TOTAL & 565 & $100 \%$ \\
\hline
\end{tabular}

Fuente: Elaboración propia

En la tabla 2 se observa que el $21 \%$ de los profesionales encuestados considera que los arbitrajes son como consecuencia de una mala definición de un contrato, y las ampliaciones de plazo, incremento de presupuesto, mayores pagos de Gastos Generales, Obras defectuosas e Inaplicación de penalidades están en un $15 \%$, que representa casi el total de las consecuencias planteadas.

Fue muy útil la encuesta ya que definió muchos aspectos actuales sobre la situación que enfrentan los profesionales que han laborado y laboran en la ejecución de obras de saneamiento administrando contratos.

De las preguntas seleccionadas a los profesionales Ingenieros se ha podido tomar conciencia de las acciones que realiza cada uno en la búsqueda del mejoramiento continuo en desarrollar nuevas formas de administración de contratos alineados a procedimientos ya estandarizados y de esta forma evitar problemas tales como arbitrajes, adicionales, ampliaciones de plazo y otros que deriven de una inadecuada formulación de cláusulas a través del contrato de obra.

De las encuestas se pudo concluir con algunos resultados dados como:

- De los 20 encuestados el 100\% son Ingenieros, un 55\% tiene más de 15 años de experiencia trabajando en obras de saneamiento.

- Un 50\% ha realizado más de 3 adicionales en una obra que el presenció.

- Un 75\% ha presenciado una ampliación de plazo máximo de 4 meses, TDR imprecisos, Inaplicación de penalidades, obras inconclusas, no renovación de cartas de fianzas, obras defectuosas, sus valores fluctúan entre los 11 al 15\% y otros parámetros están en $9 \%$, se puede concluir que la mayoría de los problemas tienen incidencia de uno $u$ otra forma en el proceso de ejecución de una obra sanitaria.

- Un $21 \%$ considera de muy relevante que los arbitrajes son consecuencias de una mala definición de las cláusulas de un contrato.

- El $100 \%$ de profesionales encuestados no conoce sobre contratos internacionales FIDIC. 
- El $90 \%$ de los profesionales encuestados cree que el no contar metodología específica para la administración de contratos estandarizados en obras de saneamiento, puede complicar el desarrollo la ejecución de la obra

- El $80 \%$ de los profesionales encuestados no cuenta con alguna metodología para la administración de contratos estandarizados.

- El $100 \%$ de los profesionales encuestados creen que contando con una administración de contratos (alineada a un modelo de estandarización), nos permitiría definir, validar y controlar el procedimiento de ejecución de la obra

- El $100 \%$ de los profesionales encuestados considera que implementando una metodología de administrar contratos estandarizados resolvería los conflictos en obras de saneamiento.

\section{PROPUESTA DE INNOVACIÓN}

\section{DESCRIPCIÓN DE LA PROPUESTA}

La propuesta del Modelo de Administración de Contratos para Mejorar la Gestión de Proyectos en Obras de Saneamiento se ha desarrollado de una manera secuencial considerando todos los grupos de procesos de gestión (inicio, planificación, ejecución, seguimiento y control, cierre).

\section{DESCRIPCIÓN DE LA ESTRUCTURA DE LA PROPUESTA}

El Inicio donde se tiene entregables que son el Perfeccionamiento del contrato estandarizado, lista de interesados, revisión de términos de referencia, bases integradas, cronograma programado y cronograma valorizado, dichos entregables de esta etapa serán generados por la Gerencia de Administración y revisadas por el Administrador de Contratos.

La Planificación donde se tiene entregables como el organigrama de roles y responsabilidades de la gerencia de proyectos, la EDT descomposición de los entregables a ser ejecutados en la administración de contratos que pueda ser controlado, los términos de referencia para el contratista también serán otorgados en este proceso para obtener el proveedor más idóneo que cumpla las competencias que el proyecto requiere.

La Ejecución es la etapa en donde se tiene a la contratista seleccionada, un contrato estandarizado firmado y aceptado de mutuo acuerdo de ambas partes y es donde el administrador de contrato en coordinación con la Supervisión tendrá que generar los entregables que se encuentran en las cláusulas del contrato estandarizado como: cumplimiento de plazos, recursos, reportes de control adicionales, deductivos, ampliaciones de plazo entre otros.

El Seguimiento y control es la etapa de revisión y gestión en la administración de contratos de los problemas y soluciones, en esta etapa se generan aprobaciones o rechazos de solicitudes de cambio (adendas al contrato), se crea documentos de check list (cumplimiento de cláusulas).

El Cierre es la etapa de cierre administrativo del contrato estandarizado, se genera los documentos (dosier) que dieron origen y fin a aprobaciones $y / 0$ rechazos de los entregables, verificación de cumplimiento, lecciones aprendidas para que posterior reinicie y continúe con la mejora continua. 


\section{DESCRIPCIÓN DE LA VIABILIDAD DE LA PROPUESTA}

Los contratos FIDIC a la fecha han sido reconocidos internacionalmente en todo el ámbito de la ingeniería y construcción, asimismo la interpretación de los contratos se encuentra reforzada con un proceso sistemático por parte de instituciones como la Cámara de Comercio Internacional y la Corte de Arbitraje Internacional de Londres.

Los modelos de contratos FIDIC principalmente el Libro Rojo (RED BOOK), contienen 20 cláusulas que cubren temas claves para la ejecución de los proyectos y la estrecha inter relación entre cliente, el ingeniero y el contratista donde destacan cláusulas como la cláusula 2, 3, 4, 6, 7, 8, 14, 15, 16, 17 y 19.

Mediante este desarrollo histórico se está logrando el perfeccionamiento de los contratos, pero a pesar de que existe una constante modificación y/o cambio es necesario la implementación de estándares internacionales las cuales este estudio de investigación propone este alineamiento a los modelos de contratos FIDIC, básicamente al Libro Rojo RED BOOK. A pesar de ello, estos contratos estandarizados no son de imperativa aplicación sino voluntaria y discrecional, de esta manera se entiende que también fueron creados a nivel internacional para ser adaptados exclusivamente a la necesidad y condiciones acorde con el país.

Para poder enmarcar se ha procedido a realizar el análisis de cláusula por cláusula tanto de los contratos estandarizados FIDIC y los contratos OSCE, teniendo como resultado la propuesta final del contrato que se detallan en las cláusulas finales modelo.

- CLÁUSULA PRIMERA: ANTECEDENTES

- Cláusula SEgUNDA: OBJETO

- ClÁusula teRCERA: BASE lEgal

- CLÁUSULA CUARTA: OBLIGACIONES DE LA ENTIDAD

- CLÁUSULA QUINTA: OBLIGACIONES DEL INSPECTOR

- CLÁUSULA SEXTO: OBLIGACIONES DE EL CONTRATISTA

- CLÁUSULA SÉPTIMA: DEL PERSONAL Y EQUIPO DE EL CONTRATISTA

- cláusula octava: De la modalidad de EJECUCIÓN DE obra y los MATERIALES a EMPLEAR

- CLÁUSULA NOVENA: DEL PLAZO DE EJECUCIÓN

- CLÁUSULA DECIMA: DEL CONTROL DE CALIDAD

- CLÁUSULA DECIMOPRIMERA: LA RECEPCIÓN DE LA OBRA

- CLÁUSUla deCIMOSEgUNDA: LA LIQUIDACIÓN DE LA OBRA

- CLÁUSULA DECIMOTERCERA: LA RESPONSABILIDAD POR DEFECTOS O VICIOS OCULTOS DE LA OBRA

- Cláusula deCIMOCUARTA: LA MEdición y VALUACIÓN DE lOS tRABajos

- CLÁUSULA DECIMOQUINTA: LAS VARIACIONES Y AJUSTES

- CLÁUSULA DECIMOSEXTA: DEL REAJUSTE

- CLÁUSULA DECIMOSÉPTIMA: DEL PRECIO CONTRACTUAL Y SU PAGO.

- CLÁUSULA DECIMOCTAVA: DE LA INTERVENCIÓN ECONÓMICA DE LA OBRA.

- CLÁUSULA DECIMONOVENA: DE LA RESOLUCIÓN DEL CONTRATO

- CLÁUSULA VIGÉSIMA: LOS RIESGOS Y RESPONSABILIDADES

- CLÁUSULA VIGÉSIMA PRIMERA: LOS SEgUROS

- CLÁUSULA VIGÉSIMA SEgUNDA: LA FUERZA MAYOR

- CLÁUSULA VIGÉSIMA TERCERA: PENALIDADES

- CLÁUSULA VIGÉSIMA CUARTA: LOS RECLAMOS Y DIVERGENCIAS Y EL ARBITRAJE

- CLÁUSULA VIGÉSIMA QUINTA: ANTICORRUPCIÓN. 
- Cláusula VIGÉSIMA SEXTA: DE NOTIFICACIONES ELECTRÓNICAS.

- ClÁUSUla VIGÉsima SÉPtiMa: MARCo legal del CONTRATO.

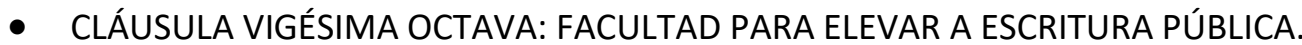

\section{DESCRIPCIÓN DE LA PROPUESTA PARA LA ADMINISTRACIÓN DEL CONTRATO.}

La Gerencia De Ingeniería. - esta Gerencia es el Área Usuaria quien tiene la responsabilidad de realizar la Nota De Pedido y los Términos de Referencia (TDR), acorde con la naturaleza de ejecución y especificaciones técnicas del proyecto previa revisión y visto bueno por la Oficina de Supervisión y Control.

La Gerencia De Administración. - A través de la División de Logística es la encargada de realizar el expediente de contratación, la convocatoria y seguimiento de la conducción del proceso de selección mediante un comité de selección, para que posteriormente se integre las bases hasta el perfeccionamiento del contrato.

La Oficina De Supervisión Y Control. - Es la encargada de velar por el cumplimiento y control de la ejecución de la obra de acuerdo al contrato establecido entre las partes.

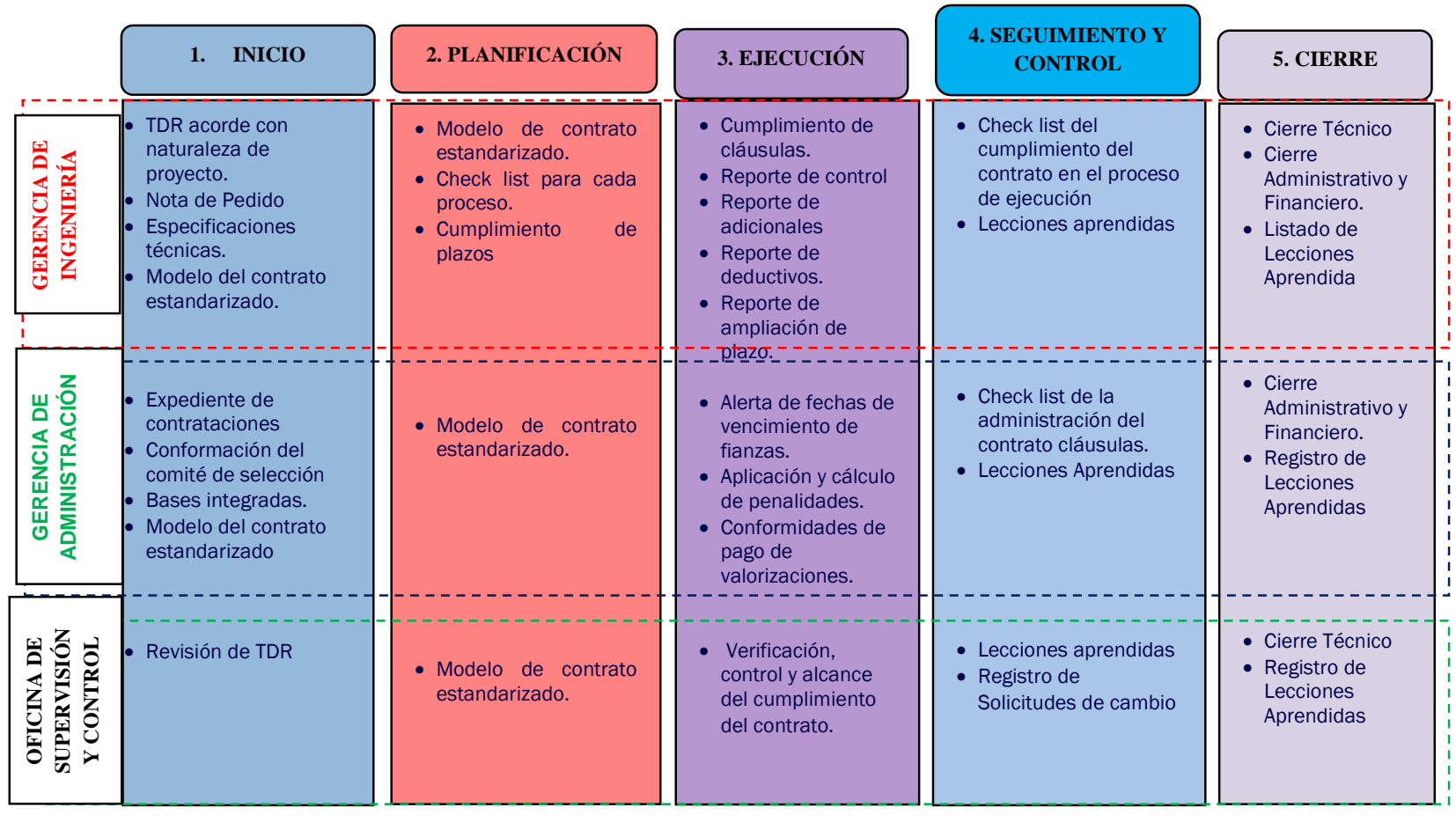

Fuente: Elaboración Propia

Figura 1 Matriz de la Administración del Contrato Estandarizado.

Teniendo el detalle de los procedimientos que presenta la matriz para la administración de contratos, tomaremos en consideración el planteamiento que debe seguir para la elaboración del check list.

\section{LOS RESULTADOS DE LA REVISIÓN DOCUMENTARIA}

Se ha procedido a revisar los contratos firmados por la entidad realizando un cuadro comparativo cláusula por cláusula adjunto a la presente donde hemos podido encontrar divergencias, ambigüedades, faltas e insuficiencias y a pesar que se ha evaluado un periodo extendido desde del 2012 - 2018 no presenta mejora continua en la elaboración de las 
cláusulas en el perfeccionamiento de los contratos muy al contrario lo básico que nos presenta el OSCE estas se ven recortadas en texto en muchos casos por los administradores de contratos.

- En todos los contratos analizados en la parte INTRODUCTORIA, no menciona que el Representante Legal esté debidamente acreditado ante Registros Públicos SUNARP, esto hace que invalide la representación para la firma del contrato en caso que el Representante Legal no acredite dicho trámite o falsifique la representatividad.

- En los ANTECEDENTES, la importancia radica en la breve descripción del rubro de cada uno de las partes, de esta forma se valida la información y queda establecido el objeto del contrato; el cual carece de descripción.

- Cuando analizamos la cláusula DEL PAGO, encontramos que las valorizaciones deberían ser explicitas indicando que serán acorde a un metrado realmente ejecutado conciliado (Contratista - Supervisión); no menciona el tiempo de entrega de la valorización ni penalidad alguna en caso de que la contratista incumpla con la entrega de la valorización mensual; asimismo, la cláusula no describe en caso de incumplimiento en la entrega de la valorización por la empresa CONTRATISTA.

- Cláusula sobre EL PLAZO DE EJECUCIÓN, existe contradicción en cuanto a la fecha de inicio de la obra por una parte menciona que el inicio se computa "desde el día siguiente de la entrega de terreno" y por otra parte menciona "el inicio de ejecución de cada obra estará supeditado y se contará a partir de la conclusión del trámite y entrega de la Resolución Administrativa", ya que el párrafo estaría recayendo en una ambigüedad para la fecha del inicio de obra, en muchos casos solo menciona que se deberá cumplir las condiciones previstas en el numeral 3.5 de la sección general de las bases donde es necesario aclarar cuáles son esas condiciones para el computo del inicio de obra o las condiciones a tratar (se notifique al contratista quien es el supervisor; que se haya hecho la entrega de terreno; que se haya hecho la entrega del expediente técnico de obra completo; que se haya otorgado el adelanto directo).

- El uso de terminología ambigua que no esclarece por ejemplo en la cláusula DECLARACIÓN DE CONTRATISTA; no precisa a que se refiere el término "incumplimiento" ya que existe muchas variables al hacer uso incumplimiento en plazo, en personal ofertado, retrasos injustificados, metrados realmente ejecutados, tiempo de ejecución, etc.

- En la cláusula de SOLUCIÓN DE CONTROVERSIAS, En caso se menciona el centro de arbitraje, es recomendable que los centros de arbitraje estén registrados y acreditados ante el OSCE, a la firma del contrato en el lugar de perfeccionamiento de este. Casi en todos los contratos estudiados no menciona como se realizará el pago por el arbitraje, además, el cumplimiento del Artículo $45^{\circ}$ de la Ley de Contrataciones del Estado, las partes pueden pactar hasta antes del inicio de la ejecución de la obra, una cláusula de solución de disputas a cargo de una: "Junta de Resolución de Disputas", siendo sus decisiones vinculantes para las partes.

- Estas incongruencias y deficiencias encontradas al momento de la firma del contrato hacen de que las obra no se puedan culminar en un 100\%, además, originan polémicas entre el contratista y la entidad razón por el cual se tienen controversias que concluyen en arbitraje y posterior paralización del proyecto de inversión; como vemos en el presente análisis de contratos periodo 2012 - 2018, actualmente dos proyectos se encuentran paralizados. 
- El presente estudio de investigación ha planteado un modelo de contrato estandarizado basado en la normativa OSCE y FIDIC tomando en consideración los estudios de las encuestas, juicio de expertos, entrevistas a expertos y otros, los cuales se resume en 28 cláusulas que contemplan y mitigan al mismo tiempo los riesgos de los problemas planteados en su totalidad.

\section{DESCRIPCIÓN DE LA FUNCIONALIDAD DE LA PROPUESTA}

- Uno de los principales factores es la comprensión completa del contrato estandarizado propuesto en el estudio de investigación por las 03 áreas propuestas (Gerencia de Ingeniería, Gerencia de Administración y Finanzas y la Oficina de Supervisión y Control).

- También es importante definir y entender claramente el papel del administrador de contrato, así como sus responsabilidades, aplicación de cláusulas, aplicación de penalidades, sistema de presentación de informes, valorizaciones, control de cambios y otros.

- Así como indica (FIDIC, 1999) una buena administración de del contrato facilita al proyecto: Mejora la relación entre las personas que compone, Examina el proceso de las relaciones contractuales con el cliente, Comprende el contrato, como leerlo, interpretarlo, y evaluarlo, Examina los aspectos claves de la administración de contratos, Es consciente de los problemas contractuales que rodea a las variaciones, retrasos, y defectos, Mantiene el proyecto dentro de las obligaciones del contrato, Aprecia los problemas de cumplimiento de contrato en la gestión del proyecto; Analiza la efectividad de los sistemas de control documental, informes, y comunicaciones; Introduce capacidad de negociación para evitar disputas con el cliente.

- Cabe mencionar que el éxito en la funcionalidad del contrato es establecer: cláusulas claras y precisas, procesos de negociación, intencionalidad de ambas partes a resolver el conflicto y por último ser coherente en la interpretación del contrato estandarizado propuesto en el estudio de investigación.

\section{CONCLUSIONES}

Se desarrolló un Modelo de Administración de Contrato Estandarizado que permitirá gestionar un proyecto de saneamiento desde la etapa inicial de la licitación, buena pro, firma de contrato, ejecución y la etapa de cierre de proyecto.

Se diseñaron formatos de aplicación para los procesos de la administración del contrato estandarizado que son el inicio, planificación, ejecución, seguimiento y control y cierre, para llevar el efectivo control del cumplimiento de cada una de las cláusulas del contrato y de esta forma que sirva como lecciones aprendidas y mejora continua.

Se diseñó un Modelo de Contrato Estandarizado con 28 cláusulas tomando en consideración las cláusulas del OSCE y cláusulas del FIDIC. Con el presente contrato estandarizado se podrá aminorar los índices de controversias, problemas de arbitrajes, ampliaciones de plazo adicionales y deductivos causales perennes en toda ejecución de obra, así mismo está diseñado para que las controversias como prioridad sean resueltas mediante conciliación preferentemente antes de iniciar un proceso de arbitraje. 
Se obtuvieron respuestas concluyentes a través de las encuestas a 20 profesionales involucrados en la administración de contratos para obras de saneamiento, donde el $100 \%$ son Ingenieros, un 55\% tiene más de 15 años de experiencia trabajando en obras de saneamiento, un $21 \%$ considera de muy relevante que los arbitrajes son consecuencias de una mala definición de las cláusulas de un contrato. El 90\% de los profesionales encuestados cree que el no contar metodología específica para la administración de contratos estandarizados en obras de saneamiento, puede complicar el desarrollo la ejecución de la obra. El $80 \%$ de los profesionales encuestados no cuenta con alguna metodología para la administración de contratos estandarizados y el $100 \%$ de los profesionales encuestados creen que contando con una administración de contratos (alineada a un modelo de estandarización), nos permitiría definir, validar y controlar el procedimiento de ejecución de la obra.

\section{RECOMENDACIONES.}

Es importante realizar la evaluación post administración de contrato, para poder monitorear si el contrato estandarizado cumplió con sus objetivos. Los formatos de gestión podrían sufrir mejoras acordes a cómo vamos viendo las lecciones aprendidas de un determinado proyecto. La administración de contratos está directamente vinculado a temas estrictamente legales para lo cual es necesario que el administrador de contrato tenga conocimiento o este asesorado por un profesional en derecho civil. Es necesario que el administrador de contratos revise y tenga amplio conocimiento del uso de los formatos planteados para las diferentes etapas, asimismo, tener experiencia en el campo como administrador de contratos.

Se recomienda se realice la validación de la propuesta modelo de contrato estandarizado, el seguimiento y control del mismo, a fin de corroborar los resultados obtenidos para la gestión de proyectos de saneamiento, el cual una vez ejecutado permitiría controlar, validar y definir el procedimiento en ejecución de obra.

\section{REFERENCIAS BIBLIOGRÁFICAS}

Almagro Y Klee. (2017). Los Contratos Internacionales De Construcción. Fidic. Estados Unidos: Wollers Kluwer.

Castillo, M. (2014). Dispute E Board En Latinoamerica: Experiencias Y Retos. Lima: F.M. Servicios Graficos.

Danos, J. (2006). El Régimen De Los Contratos Estatales En El Perú. Perú: Revista De Derecho Administrativo.

Eps Tacna S.A. (2015). Manual De Organizacion Y Funciones. Tacna: Eps Tacna S.A.

Fidic. (1999). Contratos Internacionales De Proyecto Y Construccion . Ginebra, Suiza: Fidic.

Gonzales De Cossio. (2016). La Escurridiza Noción De Arbitraje. Lima.

Guia Pmbok. (2017). Guía De Los Fundamentos Para La Direccion De Proyectos. Estados Unidos: Project Management Institute, Inc.

Jimenez, J. (2003). Tesis Doctoral Gestión De Contratos De Obras De Las Administraciones Públicas. Estudio De Los Orígenes Y Causas De Las Habituales Desviaciones Presupuestarias. Perú.

Ley De Contrataciones Del Estado. (2009). El Decreto Legislativo No. 1017. Lima: Osce.

Ministerio De Economia Y Finanzas. (2015). Guia Metodológica Para Saneamiento Basico. Lima: MefLima.

Ministerio De Economia Y Finanzas. (2018). Decreto Legislativo 1252 Modificado D.L 1432. Lima: Mef. Ministerio De Justicia. (2015). Codigo Civil . Lima: Pj. 
Organismo Supervisor De Las Contrataciones Del Estado. (2018). Reglamento Ley De Contrataciones Del Estado Ley 30225. Lima: Osce.

Paredes, G Y Gray, J. (2015). El Modelo De Resolución De Disputas Fidic (Libro Rojo) Como Alternativa Eficiente Al Actual Diseño De Solucion De Conflictos En Los Proyectos De Infraestructura Publica En Peru. Lima: Upc.

Pereira, I. Quijada, V. Pacompea G. Y Legua, M . (2010). Grupo De Trabajo De La Maestria En Contrataciones De Estado U.S.M.P - Osce. Peru.

Real Academia De La Legua Española. (2016). Diccionario De La Real Academia Española. España: Rae.

Rodriguez, M. (2006). El Contrato Internacional De Construccion. España: Revista E-Mercatoria Vol 5 $\mathrm{N}^{\circ} 01$.

Sanchez, R. (2017). Contratos Fidic: Su Importancia Dentro De La Industria De La Construcción En México. Mexico: Boletin Centro De Arbitraje.

The Joints Contracts Tribunal . (2014). Guia Juridica De La Cnudmi. Estado Unidos: Cnudmi.

Zambrano, E. (2010). Revista Contratando, Perú Contrata. Perú. 